\title{
Expansion of human SCID-repopulating cells under hypoxic conditions
}

\author{
Guénahel H. Danet, ${ }^{1}$ Yi Pan, ${ }^{2}$ Jennifer L. Luongo, ${ }^{1}$ Dominique A. Bonnet, ${ }^{3}$ \\ and M. Celeste Simon ${ }^{2}$ \\ ${ }^{1}$ Division of Hematology-Oncology, University of Pennsylvania School of Medicine, Philadelphia, Pennsylvania, USA \\ ${ }^{2}$ Howard Hughes Medical Institute and Abramson Family Cancer Research Institute, University of Pennsylvania \\ Cancer Center, Philadelphia, Pennsylvania, USA \\ ${ }^{3}$ Cancer Research UK, London Research Institute, London, United Kingdom
}

It has been proposed that bone marrow $(\mathrm{BM})$ hematopoietic stem and progenitor cells are distributed along an oxygen $\left(\mathrm{O}_{2}\right)$ gradient, where stem cells reside in the most hypoxic areas and proliferating progenitors are found in $\mathrm{O}_{2}$-rich areas. However, the effects of hypoxia on human hematopoietic stem cells (HSCs) have not been characterized. Our objective was to evaluate the functional and molecular responses of human $\mathrm{BM}$ progenitors and stem cells to hypoxic conditions. $\mathrm{BM}$ lineage-negative $\left(\mathrm{Lin}^{-}\right)$ $\mathrm{CD} 34^{+} \mathrm{CD} 38^{-}$cells were cultured in serum-free medium under $1.5 \% \mathrm{O}_{2}$ (hypoxia) or $20 \% \mathrm{O}_{2}$ (normoxia) for 4 days. Using limiting dilution analysis, we demonstrate that the absolute number of SCID-repopulating cells (SRCs) increased by 5.8 -fold in hypoxic cultures compared with normoxia, and by 4.2 -fold compared with freshly isolated $\mathrm{Lin}^{-} \mathrm{CD} 34^{+} \mathrm{CD} 38^{-}$cells. The observed increase in BM-repopulating activity was associated with a preferential expansion of $\mathrm{Lin}^{-} \mathrm{CD} 34^{+} \mathrm{CD} 38^{-}$cells. We also demonstrate that, in response to hypoxia, hypoxia-inducible factor- $1 \alpha$ protein was stabilized, surface expression of angiogenic receptors was upregulated, and VEGF secretion increased in $\mathrm{BM} \mathrm{Lin}-\mathrm{CD} 34^{+}$cultures. The use of low $\mathrm{O}_{2}$ levels to enhance the survival and/or self-renewal of human BM HSCs in vitro represents an important advance and could have valuable clinical implications.

J. Clin. Invest. 112:126-135 (2003). doi:10.1172/JCI200317669.

\section{Introduction}

The effects of hypoxia on human hematopoietic progenitors and stem cells remain poorly understood. It has only recently been reported that, in normal volunteers, the partial pressure of oxygen $\left(\mathrm{O}_{2}\right)$ and $\mathrm{O}_{2}$ saturation in human bone marrow (BM) are lower than in peripheral blood (1). The presence of hypoxic areas in the BM is determined by the architecture of medullary sinuses and the pattern of arterial blood flow in the marrow (2). It has been proposed that hematopoietic stem cells (HSCs) and progenitors are distributed along an $\mathrm{O}_{2}$ gradient, with stem cells residing in the most hypoxic areas and proliferating progenitors in $\mathrm{O}_{2}$-rich

Received for publication December 19, 2002, and accepted in revised form April 22, 2003.

Address correspondence to: Guénahel H. Danet, Division of Hematology-Oncology, University of Pennsylvania School of Medicine, 723 Biomedical Research Building-II/III, 421 Curie Boulevard, Philadelphia, Pennsylvania 19104-6160, USA.

Phone: (215) 573-3705; Fax: (215) 573-7049;

E-mail: gdanet@mail.med.upenn.edu.

Dominique A. Bonnet and M. Celeste Simon contributed equally to this work.

Conflict of interest: The authors have declared that no conflict of interest exists.

Nonstandard abbreviations used: bone marrow (BM); hematopoietic stem cell (HSC); burst-forming unit, erythroid (BFU-E); colony-forming cell (CFC); SCID-repopulating cell (SRC); bone marrow lineage-negative $\left(\mathrm{Lin}^{-}\right)$; long-term colony-initiating cell (LTC-IC); hypoxia-inducible factor-1 $\alpha$ (HIF- $1 \alpha$ ); aryl hydrocarbon receptor nuclear translocator protein (ARNT); phycoerythrin (PE); stem cell factor (SCF); VEGF receptor 2 (VEGFR2). areas (3). The data in support of this model were generated using "closed systems" in which $\mathrm{O}_{2}$ consumption by cells results in a progressive decrease in $\mathrm{O}_{2}$ level below the initial 1\%. In these conditions, Cipolleschi et al. (3) demonstrated that the ability of HSCs to repopulate the BM of lethally irradiated mice and give rise to myeloid colonies was better preserved in hypoxia $\left(\leq 1.5 \% \mathrm{O}_{2}\right.$ for 5 days $)$ than in normoxic conditions. In contrast, committed progenitors (GM-CFU) could not be preserved under the same low $\mathrm{O}_{2}$ levels. When an $\mathrm{O}_{2}$ regulation device and growth factor stimulation were used, Ivanovic et al. (4) reported a smaller expansion of mouse progenitors and a better maintenance of the number of myeloid progenitors in the recipients' BM after 8 days in hypoxia compared with normoxia. In addition, we have shown that the proliferation of embryonic hematopoietic progenitors is regulated by a hypoxia-mediated signaling pathway (5).

The available data on the effects of hypoxia on human hematopoietic cells is limited to the clonogenic activity of umbilical cord blood, mobilized peripheral blood, or BM progenitor cells. Cipolleschi et al. (6) reported an increase in burst-forming unit, erythroid (BFU-E) and a decrease in GM-CFU colonies when cord blood CD34+ cells were cultured in a 14-day clonogenic assay under severe hypoxia $\left(<1 \% \mathrm{O}_{2}\right)$. An increase in both BFU-E and GM-CFU activity in BM CD34+ cells cultured in $1.5 \% \mathrm{O}_{2}$ for 6 hours has also been reported (7). In contrast, the culture of mobilized peripheral blood CD $34^{+}$cells under severe hypoxia $\left(<1 \% \mathrm{O}_{2}\right)$ for 7 
days resulted in a decrease in the total number of colony-forming cells (CFCs) (8). Although these in vitro assays provide clues on the effect of hypoxia on the clonogenic activity of human progenitors, they may not reflect its effects on human HSCs. It is necessary to use a xenotransplantation model, such as NOD/SCID mice (9), to evaluate the functional response of human BM-repopulating cells to culture under low $\mathrm{O}_{2}$ levels. Human HSCs capable of extensive proliferation and multilineage repopulation of the BM of NOD/SCID mice are defined as SCID-repopulating cells (SRCs) $(10-12)$. SRCs are highly enriched in the BM lineage-negative $\left(\mathrm{Lin}^{-}\right) \mathrm{CD} 34^{+} \mathrm{CD} 38^{-}$fraction (10-12) and present at a higher frequency in umbilical cord blood than in adult BM $(13,14)$. We and others $(15,16-24)$ have examined various culture conditions for their ability to maintain or enhance SRC activity in vitro. From these studies, it has been shown that the ex vivo expansion of CFCs and long-term colony-initiating cells (LTC-ICs) is not necessarily associated with an increase in SRCs. Furthermore, limiting dilution analysis is required to quantitatively compare the effect of culture conditions on SRC activity $(12,17-20,24)$.

The cellular mechanisms by which human hematopoietic progenitors and stem cells respond to hypoxia have not been characterized. However, the response to hypoxia has been investigated in a variety of other cell types and models (see refs. 25-29 for reviews). The hypoxiainducible factor- $1 \alpha$ (HIF- $1 \alpha)$ protein, which is rapidly degraded under normoxic conditions, becomes stabilized (30) under low $\mathrm{O}_{2}$ levels ( $\left.<5 \%\right)$ and forms a dimer with aryl hydrocarbon receptor nuclear translocator protein (ARNT). In contrast to HIF-1 $\alpha$, ARNT levels are not regulated by hypoxia. The heterodimer HIF- $1 \alpha$-ARNT is a transcriptional activator of genes encoding for a wide variety of genes including erythropoietin, VEGF, glucose transporters, and glycolytic enzymes $(27,31)$. HIF- $2 \alpha$ $(32-34)$ and HIF-3 $\alpha$ (35) are closely related to HIF- $1 \alpha$ but have a more restricted pattern of expression and partially overlapping functions (27).

The objectives of this study were to evaluate the functional and cellular responses of human BM hematopoietic progenitors and stem cells to hypoxia. We used normal adult BM as a source of human HSCs because these cells reside in a hypoxic environment in vivo (1) and therefore may represent a more relevant model than other sources of human HSCs such as cord blood or mobilized peripheral blood. In this study, we demonstrate quantitatively that adult BM SRCs can be expanded in vitro under hypoxic conditions. This increase in BM-repopulating activity was associated with the preferential expansion of

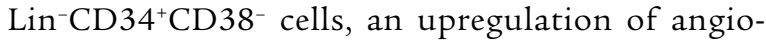
genic receptors, and an increase in VEGF production. Our results suggest that hypoxia could play a critical role in regulating the self-renewal of human $\mathrm{BM}$ HSCs and that the increase in VEGF secretions induced by hypoxia could be involved in the maintenance of HSCs in vitro.

\section{Methods}

$B M$ cell isolation. BM aspirates $(n=137)$ were obtained from healthy volunteers in accordance with the guidelines of the University of Pennsylvania Institutional Review Board for Human Subjects. At least three BM samples from three different donors were pooled for the isolation of Lin' cells. Light-density mononuclear cells were isolated using Ficoll-Paque Plus (Amersham Biosciences Corp., Piscataway, New Jersey, USA), erythrocytes were lysed using ammonium chloride, and Lincells were isolated using immunomagnetic beads (StemCell Technologies Inc., Vancouver, British Columbia, Canada). Briefly, cells were incubated with a mixture of lineage-specific antibodies (CD2, CD3, CD14, CD16, CD19, CD24, CD56, CD66b, CD41, and glycophorin A) followed by incubation with a secondary antibody conjugated to metal colloid. Cells were then eluted through a magnetized column to deplete the suspension from cells expressing lineage markers. Lin ${ }^{-}$cells were then stained with anti-human CD34-phycoerythrin (CD34$\mathrm{PE}$ ), anti-human CD38-allophycocyanine (both from Becton, Dickinson and Co., San Jose, California, USA), and propidium iodide (Molecular Probes Inc., Eugene, Oregon, USA) and sorted on a MoFlo cell sorter (Cytomation Inc., Fort Collins, Colorado, USA).

Liquid cultures. $\mathrm{BM} \mathrm{Lin}{ }^{-} \mathrm{CD} 34^{+}$or $\mathrm{Lin}^{-} \mathrm{CD} 34^{+} \mathrm{CD} 38$ cells $\left(10^{6} / \mathrm{ml}\right)$ were cultured in serum-free medium consisting of StemSpan SFEM medium (StemCell Technologies Inc.) supplemented with IL-3 $(10 \mathrm{ng} / \mathrm{ml})$, IL-6 $(10 \mathrm{ng} / \mathrm{ml})$, stem cell factor (SCF) $(300 \mathrm{ng} / \mathrm{ml})$, Flt-3 ligand $(300 \mathrm{ng} / \mathrm{ml})$, G-CSF $(50 \mathrm{ng} / \mathrm{ml}), 1 \%$ HEPES, gentamicin, and LDL $(10 \mu \mathrm{g} / \mathrm{ml})$. Cells were cultured for 4-9 days at $37^{\circ} \mathrm{C}$ in $5 \% \mathrm{CO}_{2}$-humidified incubators in normoxic $\left(20 \% \mathrm{O}_{2}\right)$ or hypoxic $\left(1.5 \% \mathrm{O}_{2}\right)$ conditions. Hypoxic cultures were performed in a two-gas incubator (Jouan Inc., Winchester, Virginia, USA) equipped with an $\mathrm{O}_{2}$ probe to regulate $\mathrm{N}_{2}$ levels. In some experiments, normoxic cultures of $\mathrm{BM} \mathrm{Lin}{ }^{-} \mathrm{CD} 34^{+}$cells were supplemented with human VEGF $(100 \mathrm{ng} / \mathrm{ml})$, angiopoietin-1 $(100 \mathrm{ng} / \mathrm{ml})$, or angiopoietin-2 (100 $\mathrm{ng} / \mathrm{ml}$ ), all obtained from R\&D Systems Inc. (Minneapolis, Minnesota, USA).

Phenotypic analysis and cell division tracking. $\mathrm{Lin}^{-} \mathrm{CD} 34^{+}$ cells were harvested after 4 days of culture in hypoxic $\left(1.5 \% \mathrm{O}_{2}\right)$ or normoxic conditions. Cell number and viability were evaluated using trypan blue exclusion. Cells were incubated with anti-human CD34 and the following antibodies: CD31, CD38, CD117 (c-kit), and HLA-DR (Becton, Dickinson and Co.), CD90 from Beckman Coulter (Miami, Florida, USA), CD133 from Miltenyi Biotec (Auburn, California, USA), CD135 and CD162 from Immunotech Inc. (Westbrook, Maine, USA), CXCR4, Tie-1, Tie-2, Flt-1, and VEGF receptor 2 (VEGFR2, also known as Kdr, Flk-1) from R\&D Systems Inc., and vWF from Santa Cruz Biotechnology Inc. (Santa Cruz, California, USA). In some experiments, freshly isolated $\mathrm{Lin}^{-} \mathrm{CD} 34^{+}$cells were labeled with CFSE (Molecular Probes Inc., Eugene, Oregon, USA) as previously described (15). Cells were cultured 
for 4 days in hypoxic $\left(1.5 \% \mathrm{O}_{2}\right)$ or normoxic conditions and stained for CD34-allophycocyanin and either the appropriate isotype control or one of the following antihuman antibodies: CD38, CD90, CD117, CD133, CD135, or HLA-DR. Cells were then analyzed on a FACSCalibur flow cytometer (Becton, Dickinson and Co.).

Cell cycle analysis. $\mathrm{Lin}^{-} \mathrm{CD} 34^{+}$cells cultured for 4 days in hypoxic $\left(1.5 \% \mathrm{O}_{2}\right)$ or normoxic conditions were harvested and cell cycle analysis was performed as previously described (36). Briefly, cells were incubated with CD34-PE, then fixed with a $0.4 \%$ formaldehydebuffered solution, washed, and permeabilized with a $0.2 \%$ Triton X-100 solution on ice. After two washes in PBS plus 2\% FBS, cells were labeled with Ki-67-FITC (Becton, Dickinson and Co.). Finally, each sample was washed twice and resuspended in a $10 \mu \mathrm{M}$ solution of DAPI (Molecular Probes Inc.) in PBS plus 2\% FBS. Cells were analyzed using a Becton-Dickinson LSR flow cytometer equipped with a $325-\mathrm{nm}$ helium-cadmium UV laser and a 488-nm argon-ion laser.

CFC and LTC-IC assays. Human CFCs were assayed in semisolid methylcellulose medium in standard conditions (12). LTC-IC cultures were established on preformed M210B4 stroma layers using human myeloid long-term culture medium (MyeloCult 5100; StemCell Technologies Inc.) according to described methods (37, 38). Limiting dilutions (at least three replicates/dilution) were performed in 96-well plates in which 500-2,000 Lin ${ }^{-} \mathrm{CD} 34^{+} \mathrm{CD} 38^{-}$cells per well were plated. After 5 weeks in coculture with stroma cells, the content of each well was transferred in methylcellulose medium to reveal CFC activity.

Transplantation and analysis of NOD/SCID mice. Eightweek-old sublethally irradiated ( $275 \mathrm{cGy}$ at $240 \mathrm{cGy} / \mathrm{min}$ ) $\mathrm{NOD} / \mathrm{LtSz}$-scid (NOD/SCID) mice were transplanted with fresh or cultured $\mathrm{BM} \mathrm{Lin}{ }^{-} \mathrm{CD} 34^{+} \mathrm{CD} 38^{-}$cells by lateral tail vein injection according to a standard protocol (12). Cultured $\mathrm{Lin}^{-} \mathrm{CD} 34^{+} \mathrm{CD} 38^{-}$cells were not resorted for this phenotype prior to transplantation. Lin ${ }^{-} \mathrm{CD} 34^{+}{ }^{+} \mathrm{CD} 38^{-}$cells cultured for $0,4,6$, and 9 days in hypoxic or normoxic conditions were injected into mice at doses ranging from 300 to 40,000 cells per mouse (at least three mice per cell dose per experiment). Lin ${ }^{-} \mathrm{CD} 34^{+} \mathrm{CD} 38^{-}$cells were coinjected with 0.5 million to 1 million accessory cells consisting of irradiated $(1,500$ cGy) BM or cord blood mononuclear or lineage-positive cells as previously described (39). Mice were sacrificed 8-10 weeks after transplant and BM from the femurs, tibiae, and iliac crests of each mouse were harvested. To prepare mouse BM cells for flow cytometry, BM cells were incubated with a $6 \%$ ammonium chloride solution, then washed and incubated with anti-human CD45-FITC, CD33-PE, (Beckman Coulter), and CD19-allophycocyanin (Becton, Dickinson, and Co.), and propidium iodide. For each group of mice analyzed, an aliquot of cells was also stained with matching isotype controls. For each sample, 100,000 scatter- and live-gated cells were acquired to determine engraftment. Transplanted mice showing at least $0.1 \%$ human $\mathrm{CD}^{+} 5^{+}$cells and both human myeloid $\left(\mathrm{CD} 33^{+}\right)$and lymphoid $\left(\mathrm{CD} 19^{+}\right)$cells were considered engrafted, as shown in Figure 3d.

RT-PCR. RNA was isolated from freshly purified $\mathrm{Lin}^{-} \mathrm{CD} 34^{+} \mathrm{CD}^{-} 8^{-}$or $\mathrm{Lin}^{-} \mathrm{CD} 34^{-} \mathrm{CD} 38^{-}$cells using the RNAqueous isolation kit (Ambion Inc., Austin, Texas, USA). RNA extracted from K562 cells was used as a positive control for the PCR reactions. Standard reverse transcription was done using SuperScript II reverse transcriptase (Invitrogen Corp., San Diego, California, USA). The primers used were HIF-1 $\alpha$ : 5'-AAGTCTCGAGATGCAGCCAGA and 5'-AGTTAGTTCAAA CTGACTTAATCC; HIF-2 $\alpha$ : $5^{\prime}$-GCCCCTGCTGTCCTGCCTCATCA and $5^{\prime}$-ATCCGTCTGGGTACTGCATTGGTCCTT; and ARNT: 5'AGGAATAGTGGCCTAGCCCCT and 5'-ATTGTTGTAGCTGTTGCTCTG. PCR was done using the Advantage-GC cDNA PCR kit (Clontech Laboratories Inc., Palo Alto, California, USA). The amplification cycles were: $94^{\circ} \mathrm{C}$ for 4 minutes followed by 35 cycles of $94^{\circ} \mathrm{C}$ for 10 minutes $+58^{\circ} \mathrm{C}$ for 30 seconds $+72^{\circ} \mathrm{C}$ for 2 minutes, and a final extension step at $72^{\circ} \mathrm{C}$ for 10 minutes was included before samples were cooled at $4^{\circ} \mathrm{C}$.

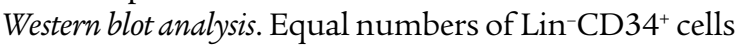
were cultured overnight in serum-free conditions $\left(10^{6} / \mathrm{ml}\right)$ at $1.5 \% \mathrm{O}_{2}$ in a hypoxic workstation $\left(\right.$ Invivo $_{2}$; Ruskinn Technology, Leeds, United Kingdom) or in normoxic conditions. Cells were directly lysed in $1 \times$ SDS loading buffer and boiled at $95^{\circ} \mathrm{C}$ for 10 minutes. The mouse anti-HIF- $1 \alpha$ antibody used was from Becton, Dickinson and Co. (catalog no. H72320) and the rabbit anti-ARNT antibody was from Novus Biologicals Inc. (catalog no. NB 100-110; Littleton, Colorado, USA).

Human VEGF ELISA and lactate assay. Conditioned media from overnight cultures $(n=5)$ of $\mathrm{Lin}^{-} \mathrm{CD} 34^{+}$ cells were harvested and human VEGF and erythropoietin concentrations were determined by ELISA (R\&D Systems Inc.) according to the manufacturer's procedure. Lactate concentrations in the conditioned media were also determined after overnight culture in hypoxic $\left(1.5 \% \mathrm{O}_{2}\right)$ and normoxic conditions using a colorimetric assay (Sigma-Aldrich, St. Louis, Missouri, USA) based on the enzymatic conversion of lactate to pyruvate and $\mathrm{H}_{2} \mathrm{O}_{2}$ by lactate oxidase.

Statistics. Data are presented as mean \pm SD or mean \pm SEM. Statistical differences were evaluated using the Student $t$ test. In the limiting dilution analysis used to determine SRC frequencies, mice with at least $0.1 \%$ human cells were considered engrafted. The data from limiting dilution experiments were analyzed using the single-hit Poisson model, and SRC frequencies were determined using the maximum likelihood estimator as previously shown $(12,13,16-20,24)$. We used $\chi^{2}$ analysis to verify the internal consistency of our data and validate the use of Poisson statistics.

\section{Results}

Preferential expansion of $\mathrm{Lin}^{-} \mathrm{CD} 34^{+} \mathrm{CD} 38^{-}$cells in hypoxia. $\mathrm{Lin}^{-} \mathrm{CD} 34^{+}$and $\mathrm{Lin}^{-} \mathrm{CD} 34^{+} \mathrm{CD} 38^{-}$cells were cultured in serum-free conditions for 4 days under normoxia $(20 \%$ $\left.\mathrm{O}_{2}\right)$ or hypoxia $\left(1.5 \% \mathrm{O}_{2}\right)$. We used this level of $\mathrm{O}_{2}$ based 
on previous reports indicating that mouse BM-repopulating cells can be maintained when cultured under $1 \% \mathrm{O}_{2}(3,4)$. As shown in Figure 1a, the level of expansion of $\mathrm{Lin}^{-} \mathrm{CD}_{3} 4^{+}$cells was decreased in hypoxia compared with normoxia. In contrast, the expansion of $\mathrm{Lin}^{-} \mathrm{CD} 34^{+} \mathrm{CD} 38^{-}$cells, a subpopulation enriched in primitive progenitors and stem cells, was greater in hypoxic conditions than in normoxia (2.4-fold vs. 1.5 fold, respectively). The differential effect of hypoxia on subsets of $\mathrm{Lin}^{-} \mathrm{CD} 34^{+}$cells was further investigated using CFSE to track the division history of each primitive subset $\left(\mathrm{CD}^{+}{ }^{+}, \mathrm{HLA}-\mathrm{DR}^{-}, \mathrm{CXCR}^{+}, \mathrm{CD} 117^{\text {low }}\right.$, $\mathrm{CD}_{133}{ }^{+}, \mathrm{CD} 135^{+}$) during the 4-day culture (Figure 1b). Overall, CFSE profiles indicated that all examined subsets of $\mathrm{Lin}^{-} \mathrm{CD} 34^{+}$cells cultured in hypoxia divided at a slower rate than in normoxia. In particular, HLA-DRcells divided only once or twice during the 4-day culture period. Despite this general slow proliferative rate, a small fraction (4-18\%) of the cells in each subset was associated with a lower CFSE fluorescence, indicative of a rapid rate of division. These results show that a small subset of primitive BM CD $34^{+}$cells can rapidly proliferate under low $\mathrm{O}_{2}$ levels while most $\mathrm{CD}_{3} 4^{+}$cells have a decreased rate of division. This suggests that $\mathrm{O}_{2}$ levels can differentially regulate the proliferation of primitive subsets of human BM cells.

Hypoxia regulates the cell cycle of $\mathrm{Lin}^{-} \mathrm{CD} 34^{+}$cells. We evaluated the effect of hypoxia on the cell cycle of $\mathrm{Lin}^{-} \mathrm{CD} 34^{+}$cells after 4 days of culture in hypoxic or normoxic conditions. Before culture, less than $1 \%$ of sorted $\mathrm{Lin}^{-} \mathrm{CD} 34^{+}$cells was found in the $\mathrm{S}$ or $\mathrm{G}_{2} / \mathrm{M}$ fractions (data not shown). After 4 days under hypoxia, $34.2 \% \pm 3.5 \%$ of $\mathrm{CD}_{3} 4^{+}$cells was found in $\mathrm{S}+\mathrm{G}_{2} / \mathrm{M}$ phases compared with $46.3 \% \pm 3.4 \%$ in normoxia (Table 1). A similar trend was observed for $\mathrm{Lin}^{-} \mathrm{CD} 34^{-}$cells (data not shown). These results indicate that the slower rate of expansion of $\mathrm{Lin}^{-} \mathrm{CD} 34^{+}$cells under hypoxia is at least partially due to slower cell cycling and fewer cell divisions (as shown by DAPI and CFSE, respectively). We also examined the effect of hypoxia on the transition from $G_{0}$ to $G_{1}$ phase using Ki-67 to distinguish cells in $\mathrm{G}_{0}$ phase $\left(\mathrm{Ki}-67^{-}\right)$from those in $\mathrm{G}_{1}$ phase $\left(\mathrm{Ki}-67^{+}\right)$. Table 1 shows that, in normoxic conditions, the $G_{0} / G_{1}$ ratio (\% cells in $G_{0}$ to \% cells in $G_{1}$ ) was $87 \pm 12$, indicating that most cells found in the $G_{0}+G_{1}$ peak (as defined by DAPI staining) were resting. In hypoxic conditions, the $G_{0} / G_{1}$ ratio was only $13 \pm 3$ due to a 6.7 fold increase in cells in $G_{1}$ phase and fewer cells in $G_{0}$ phase. These results suggest that hypoxia preferentially promotes the transition of $\mathrm{Lin}^{-} \mathrm{CD} 34^{+}$cells from $\mathrm{G}_{0}$ to $G_{1}$ and/or induces a cell cycle arrest in $G_{1}$ phase.

Effects of hypoxia on CFCs and LTC-ICs. We evaluated the clonogenic activity of $\mathrm{Lin}^{-} \mathrm{CD} 34^{+}$cells after 4 days of culture in hypoxic or normoxic conditions (Figure 2). After 4 days of culture, the total number of CFCs was increased by twofold compared with freshly isolated $\mathrm{Lin}^{-} \mathrm{CD} 34^{+}$cells (Figure $2 \mathrm{a}$ ). The level of $\mathrm{O}_{2}$ had no significant effect on lineage-committed progenitors, with the exception of granulocytic colonies (G-CFU), which decreased in number under hypoxic conditions. We also examined the effect of hypoxia on more primitive progenitors. LTC-ICs were equally expanded (1.4-fold) after 4 days of culture in hypoxia or normoxia (Figure 2b). In normoxia, only LTC-IC granulocyte-macrophage colonies significantly increased while, in hypoxia, both granulocyte-macrophage and erythroid colonies

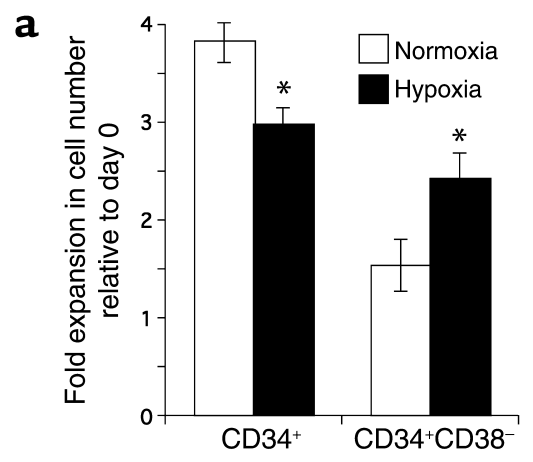

b $\operatorname{Lin}^{-} \mathrm{CD} 34^{+}$

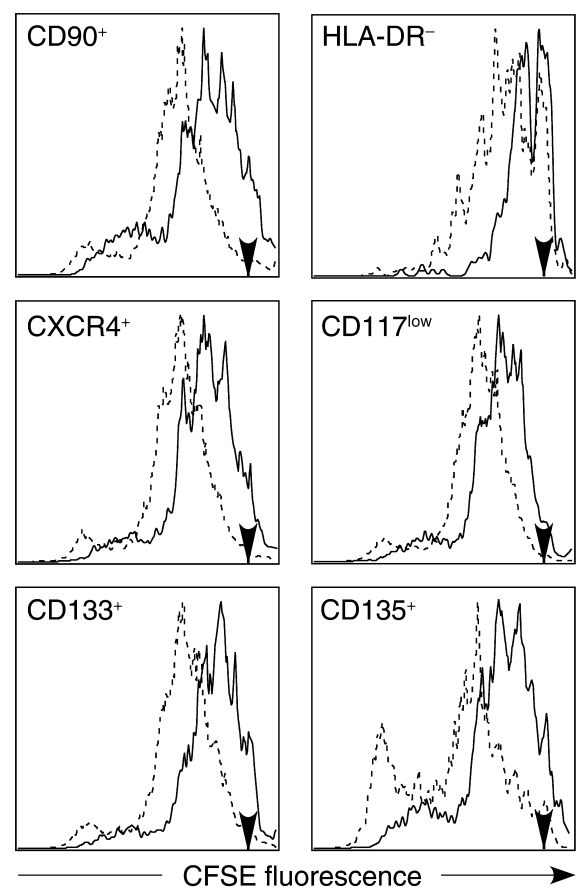

\section{Figure 1}

Expansion and division history of BM cells after 4 days in culture in

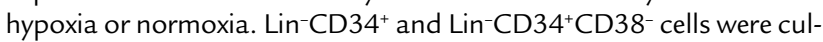
tured in serum-free conditions for 4 days in the presence of IL-3, IL-6, SCF, Flt-3 ligand, and G-CSF. (a) Expansion. The fold increase in cell number relative to the initial cell number plated (day 0 ) is represented for each subpopulation cultured in normoxia (white bars) or hypoxia (black bars). ${ }^{*} P<0.05$. (b) Division history of primitive subpopulations of $\mathrm{Lin}^{-} \mathrm{CD} 34^{+}$cells. Freshly isolated $\mathrm{Lin}^{-} \mathrm{CD} 34^{+}$cells were labeled with CFSE, cultured for 4 days in hypoxia $\left(1.5 \% \mathrm{O}_{2}\right)$ or normoxia, and analyzed for CFSE fluorescence intensity and expression of markers associated with primitive progenitors and stem cells. Histograms (representative of three separate experiments) of CFSE fluorescence in various $\mathrm{Lin}^{-} \mathrm{CD} 34^{+}$cell subsets are shown after 4 days of culture in hypoxia (solid lines) or normoxia (dashed lines). Day 0 CFSE fluorescence intensity is indicated by an arrow on each histogram. 
Table 1

Cell cycle analysis of BM Lin-CD34+ cells after 4 days of culture in hypoxia or normoxia

\begin{tabular}{lcc} 
& \multicolumn{2}{c}{ Lin-CD34 $^{+}$} \\
& Hypoxia & Normoxia \\
$\% \mathrm{G}_{0}+\mathrm{G}_{1}$ & $65.8 \pm 3.1$ & $53.7 \pm 4.0$ \\
$\mathrm{G}_{0} / \mathrm{G}_{1}$ ratio & $13 \pm 3$ & $87 \pm 12$ \\
$\% \mathrm{~S}$ & $25.8 \pm 2.3$ & $34.2 \pm 2.9$ \\
$\% \mathrm{G}_{2}+\mathrm{M}$ & $8.4 \pm 1.2$ & $12.1 \pm 0.5$
\end{tabular}

$\mathrm{BM} \mathrm{Lin-CD} 34^{+}$cells were sorted at day 0 and analyzed by flow cytometry after 4 days in culture under hypoxia $\left(1.5 \% \mathrm{O}_{2}\right)$ or normoxia. Cells were stained with $\mathrm{CD} 34, \mathrm{Ki}-67$, and DAPI. Values represent mean \pm SD of three experiments.

increased. This increase in BFU-E was the only hypoxia-specific effect on primitive progenitors we observed. These results indicate that a 4-day culture in hypoxia has a very limited effect on both committed and primitive progenitors.

SCID-repopulating activity in cells cultured under bypoxic conditions. First, we evaluated the effect of hypoxia on the SCID-repopulating ability of $\mathrm{BM} \mathrm{Lin}-\mathrm{CD} 34^{+} \mathrm{CD} 38^{-}$cells cultured for 4, 6, and 9 days in the presence of IL-3, IL-6, SCF, Flt-3 ligand, and G-CSF. In these conditions, SRCs could be maintained for only 4 days in culture, regardless of $\mathrm{O}_{2}$ levels. No human cells were detectable in the $\mathrm{BM}$ of mice $(n=16)$ transplanted with cells cultured for 6 or 9 days. These results indicate that in our conditions, hypoxia did not delay the loss of BM-repopulating activity observed after 4 days in normoxic cultures.

We used a limiting dilution analysis to quantitatively evaluate the effect of hypoxia on SCID-repopulating cells after 4 days. For this purpose, increasing numbers (ranging from 300 to 20,000) of fresh BM $\mathrm{Lin}^{-} \mathrm{CD} 34^{+} \mathrm{CD} 38^{-}$cells or cells cultured under hypoxia or normoxia for 4 days were transplanted at increasing doses into NOD/SCID mice (Figure 3). For each experimental condition (day 0 , day 4 hypoxia, and day 4 normoxia), we estimated the number of $\mathrm{Lin}^{-} \mathrm{CD} 34^{+} \mathrm{CD} 38$ cells required to statistically inject one SRC per mouse $(12,13,20)$. Table 2 shows the frequencies of engrafted mice for each cell dose used in each experimental condition. We estimated that the frequency of SRCs in freshly purified $\mathrm{Lin}^{-} \mathrm{CD} 34^{+} \mathrm{CD} 38^{-}$cells (Table 2 ) was 1 in 1,009. After 4 days of culture, we determined that the frequency of SRCs in hypoxic conditions ( 1 in 577 Lin $^{-} \mathrm{CD} 34^{+} \mathrm{CD} 38^{-}$cells) was significantly higher $(P=0.0017)$ than in normoxic cultures (1 SRC in 2,108 $\mathrm{Lin}^{-} \mathrm{CD} 34^{+} \mathrm{CD} 38^{-}$cells). Consequently, we observed a 3.6-fold increase in SRC frequency in hypoxic cultures compared with normoxia. In contrast, the apparent increase in SRC frequency between freshly isolated Lin $^{-} \mathrm{CD} 34^{+} \mathrm{CD}^{-} 8^{-}$cells (1 SRC in 1,009 cells) and day 4 hypoxic cultures ( 1 in 577 cells) was not significant $(P=0.098)$. However, taking into account the expansion of $\mathrm{Lin}^{-} \mathrm{CD} 34^{+} \mathrm{CD} 38^{-}$CFCs after 4 days (Figure 1a), we estimate that in hypoxia, the absolute number of SRCs increased by 5.8 -fold compared with the number in normoxia, and by 4.2 -fold compared with

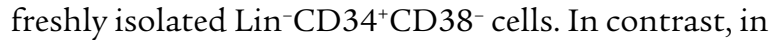
normoxic conditions, the absolute number of SRCs was just maintained in comparison with freshly isolated $\mathrm{Lin}^{-} \mathrm{CD} 34^{+} \mathrm{CD} 38^{-}$cells. In this study, we did not formally assess the effect of hypoxia on the selfrenewal of human HSCs since no secondary transplants were performed. However, our results clearly demonstrate that low $\mathrm{O}_{2}$ levels can substantially increase the number of BM-repopulating cells compared with normoxic conditions.

Molecular response of $\mathrm{Lin}^{-} \mathrm{CD} 34^{+} \mathrm{CD} 38^{-}$cells to bypoxia. We examined whether human BM progenitors and stem cells express the critical components of the cellular response to hypoxia, such as HIF-1 $\alpha$ and ARNT. Figure 4a shows that both HIF-1 $\alpha$ and ARNT are constitutively expressed in purified $\mathrm{Lin}^{-} \mathrm{CD} 34^{+} \mathrm{CD} 38^{-}$cells. HIF- $2 \alpha$ expression could not be detected in $\mathrm{Lin}^{-} \mathrm{CD} 34^{+} \mathrm{CD} 38^{-}$cells but was present at very low levels in $\mathrm{Lin}^{-} \mathrm{CD} 34^{-} \mathrm{CD} 38^{-}$cells, a subpopulation enriched in CD34- SRCs (data not shown). Since HIF- $\alpha$ subunits are regulated at the protein level,
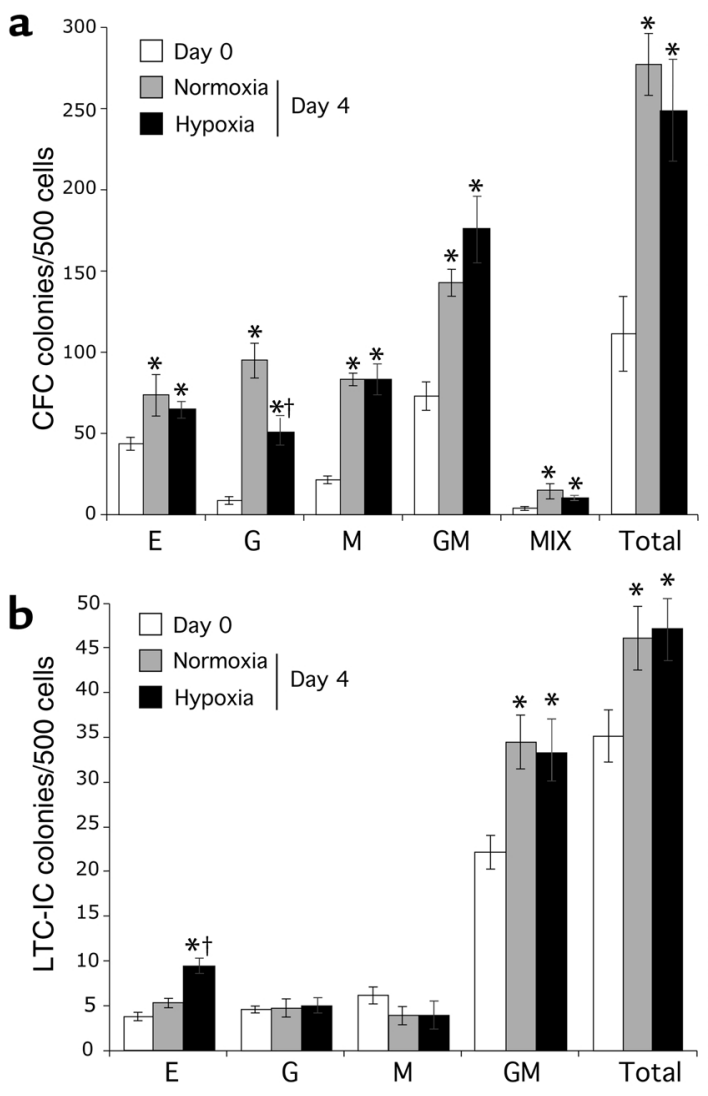

Figure 2

Effect of hypoxia on BM progenitor activity. The CFC (a) and LTC-IC (b) activity of BM Lin- ${ }^{-} D 34^{+} \mathrm{CD} 38^{-}$cells was evaluated before (day 0 ; white bars) and after culture for 4 days under normoxic (gray bars) or hypoxic $\left(1.5 \% \mathrm{O}_{2}\right.$; black bars) conditions. Data shown represent the number (mean $\pm S D$ ) of BFU-E (E), granulocyte $(G)$, monocyte $(M)$, granulocyte-monocyte (GM), mixed (MIX, i.e., GM colonies with erythroid cells), and total number of colonies from three separate experiments. The paired Student $t$ test was performed to compare day 0 with day 4 cells $\left({ }^{*} P<0.05\right)$ and to compare hypoxic to normoxic conditions after 4 days of culture $\left({ }^{\dagger} P<0.05\right)$. 

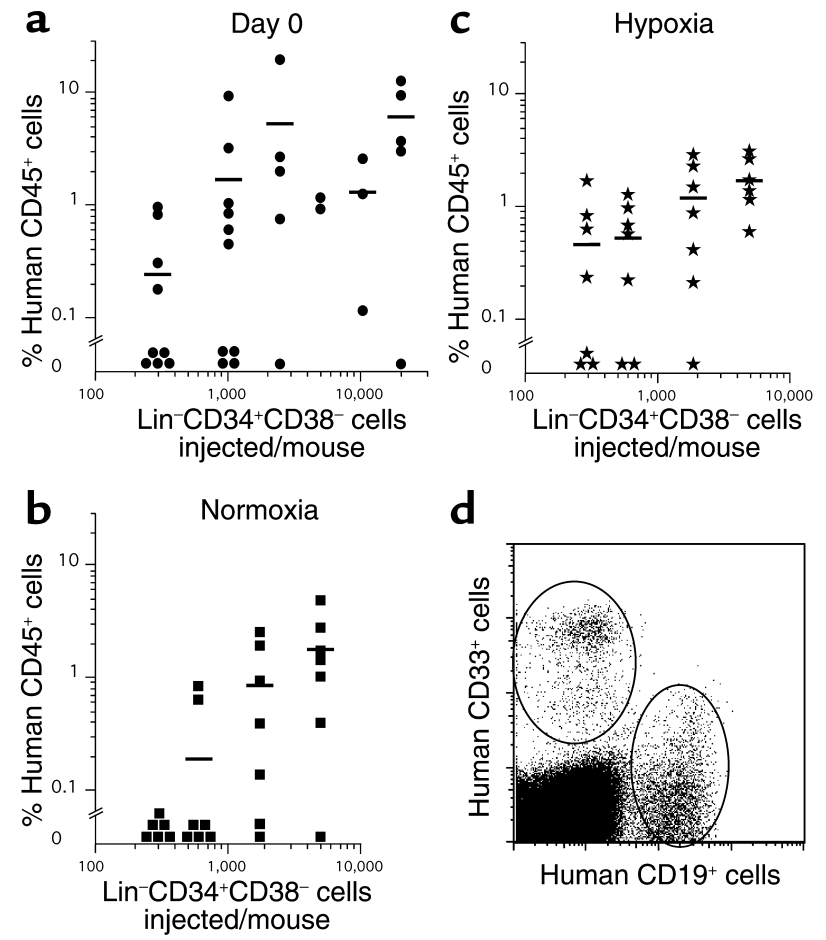

Figure 3

Quantitative analysis of the effect of hypoxia on SRC frequency. The frequency of SRCs in freshly isolated $\mathrm{Lin}^{-} \mathrm{CD} 34^{+} \mathrm{CD} 38^{-}$cells (a, day $0)$ or cells cultured for 4 days under normoxic (b) or hypoxic $(1.5 \%$ $\mathrm{O}_{2}$ ) (c) conditions was determined by limiting dilution analysis. For each NOD/SCID mouse $(n=88)$, the level of human engraftment (percentage of $\mathrm{CD} 45^{+}$cells) is represented as a function of the number of $\mathrm{Lin}^{-} \mathrm{CD} 34^{+} \mathrm{CD} 38^{-}$cells transplanted. (d) Representative flow cytometry dot plot showing the presence of both human myeloid $\left(\mathrm{CD}_{3}{ }^{+}\right)$and lymphoid $\left(\mathrm{CD} 19^{+}\right)$cells in NOD/SCID BM 6-8 weeks after transplantation.

we evaluated the effect of hypoxia and normoxia on HIF- $1 \alpha$ protein. Figure $4 b$ shows that HIF- $1 \alpha$ protein can be detected in cells cultured in hypoxia but not under normal $\mathrm{O}_{2}$ levels. In contrast, ARNT protein levels were unaffected by $\mathrm{O}_{2}$ levels. These results demonstrate that HIF can mediate the hypoxic response of human BM progenitors and stem cells.

Hypoxia regulates expression of angiogenic factors in $\mathrm{Lin}^{-} \mathrm{CD} 34^{+}$cells. Angiogenic factors and receptors such as VEGF and Flt- 1 are regulated by $\mathrm{O}_{2}$ levels (27). VEGF has been implicated in the regulation of HSC survival (40) and recruitment (41). To investigate whether the increase in SRC activity observed under hypoxia was associated with changes in the expression of angiogenic molecules, $\mathrm{Lin}^{-} \mathrm{CD} 34^{+}$cells cultured for 4 days at $1.5 \% \mathrm{O}_{2}$ or at normoxia were analyzed by flow cytometry for cell surface expression of VEGF receptor 1 (VEGFR1, also known as Flt-1), VEGFR2, Tie-1, Tie-2, and vWF. Levels of VEGF cell surface receptors (VEGFR1 and VEGFR2) increased slightly, whereas angiopoietin receptors (Tie-1 and Tie-2) were more clearly upregulated in cells cultured in hypoxic conditions (Figure 5a). In contrast, c-kit and Flt-3 lig- and were unchanged, and $\mathrm{vWF}$ and MHC class II (HLA-DR) expression was downregulated under the same conditions (Figure 5a). It is unlikely that the upregulation of VEGFR1, VEGFR2, Tie-1, and Tie-2 was due to a nonspecific response to low $\mathrm{O}_{2}$ levels since other surface molecules were either unchanged or downregulated. These results suggest that human progenitors and stem cells can specifically enhance their responsiveness to angiogenic factors under hypoxic conditions.

Hypoxia-regulated genes include VEGF $(42-44)$, erythropoietin (45), and glycolytic enzymes $(46,47)$ such as lactate dehydrogenase (48). To determine whether these HIF targets are upregulated in BM progenitors and stem cells, we cultured $\mathrm{Lin}^{-} \mathrm{CD} 34^{+}$cells for 18 hours under normoxic or hypoxic conditions and assayed VEGF, erythropoietin, and lactate concentrations in conditioned medium. Figure $5 \mathrm{~b}$ shows that both VEGF and lactate secretions were increased by more than twofold, demonstrating that hypoxia upregulates VEGF production and glycolytic enzyme activity in human BM progenitors and stem cells. In contrast, erythropoietin was undetectable in the medium after 18 hours of culture regardless of $\mathrm{O}_{2}$ levels, suggesting that HIF targets may be differentially regulated in BM cells. Our results indicate that the hypoxic response of human progenitors and HSCs is characterized by a rapid increase in VEGF secretion and glycolytic activity. This rapid increase in VEGF secretion may play a critical role in the survival and expansion of human BM-repopulating cells under hypoxia.

To determine whether angiogenic cytokines play a role in the upregulation of angiogenic receptors observed under hypoxic conditions, $\mathrm{Lin}^{-} \mathrm{CD} 34^{+}$cells were cultured in normoxia in the presence of VEGF, angiopoietin-1, or angiopoietin-2 for 4 days. Interestingly, VEGFR2 surface expression was enhanced to levels comparable to those observed under hypoxia when cells were cultured in the presence of either VEGF,

\section{Table 2}

Frequency of NOD/SCID engraftment observed with increasing doses of fresh or cultured Lin-CD34+CD38- cells after 4 days of culture in hypoxia or normoxia

No. of NOD/SCID mice engrafted/transplanted Day 0

No. injected cells

300

600

1,000

1,800

2,500

$\geq 5,000$

$4 / 9(44 \%)$
ND
$6 / 10(60 \%)$
ND
$4 / 5(80 \%)$
$9 / 10(90 \%)$
1 in 1,009

$\begin{array}{cc}\text { Normoxia } & \text { Hypoxia } \\ 0 / 6(0 \%) & 4 / 7(57 \%) \\ 2 / 7(28 \%) & 5 / 7(71 \%) \\ \text { ND } & \text { ND } \\ 5 / 7(71 \%) & 6 / 7(85 \%) \\ \text { ND } & \text { ND } \\ 6 / 7(85 \%) & 6 / 6(100 \%) \\ 1 \text { in } 2,108^{A} & 1 \text { in } 577^{\mathrm{B}}\end{array}$

NOD/SCID mice $(n=88)$ were injected with increasing doses of BM $\mathrm{Lin}^{-} \mathrm{CD} 34^{+} \mathrm{CD} 38^{-}$cells and analyzed 8-10 weeks after transplantation. Mice with $\mathrm{BM}$ containing at least $0.1 \%$ human myeloid $\left(\mathrm{CD} 33^{+}\right)$and lymphoid $\left(\mathrm{CD} 19^{+}\right)$cells were considered engrafted. ASignificantly different from day 0 $(P=0.04)$. ${ }^{B}$ Significantly different from day 4 normoxia $(P=0.0017)$. 
$\mathbf{a}$
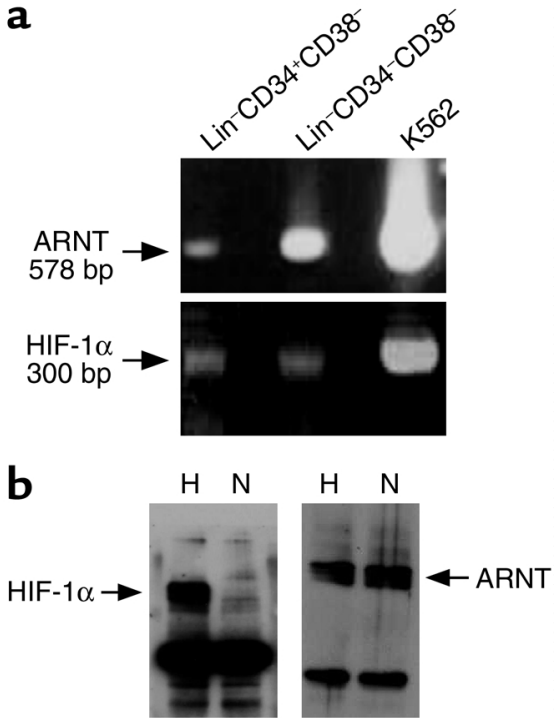

\section{Figure 4}

Expression of HIF in BM progenitors and stem cells. (a) RT-PCR analysis of ARNT and HIF- $1 \alpha$ performed on RNA extracted from BM $\mathrm{Lin}^{-} \mathrm{CD} 34^{+} \mathrm{CD} 38^{-}$, $\mathrm{Lin}^{-} \mathrm{CD} 34^{-} \mathrm{CD} 38^{-}$, and $\mathrm{K} 562$ cells (as a positive control). (b) Western blot analysis of cell extracts from BM $\mathrm{Lin}^{-} \mathrm{CD} 34^{+}$cells cultured overnight under hypoxic $(\mathrm{H})$ or normoxic $(\mathrm{N})$ conditions using antibodies against ARNT and HIF-1 $\alpha$. All gels are representative of at least three experiments.

angiopoietin-1, or angiopoietin-2 (Figure 5c). In contrast, these cytokines had no effect on the expression or Flt-1, Tie-1, or Tie-2 (data not shown).

\section{Discussion}

In this report, we demonstrate for the first time that SRCs from human $\mathrm{BM}$ can be expanded in vitro under hypoxic conditions. We used $\mathrm{BM} \mathrm{Lin}{ }^{-} \mathrm{CD} 34^{+} \mathrm{CD} 38^{-}$ cells, a population highly enriched in SRCs (12), and limiting dilution analysis to quantitatively evaluate the effect of hypoxia on SRCs. First, we determined that the frequency of SRCs in fresh adult BM is 1 in 1,009 $\mathrm{Lin}^{-} \mathrm{CD} 34^{+} \mathrm{CD} 38^{-}$cells. This result demonstrates that the frequency of SRCs in adult BM is only half of the SRC frequency that was reported previously for cord blood ( 1 in 617 Lin $^{-} \mathrm{CD} 34^{+} \mathrm{CD} 38^{-}$cells) (12). This is consistent with other reports indicating that the frequency of SRC is higher in cord blood than in BM or mobilized peripheral blood $(13,14)$. To evaluate the effect of hypoxia on BM SRCs in vitro, we used culture conditions (IL-3, IL-6, SCF, Flt-3 ligand, and G-CSF in serum-free medium) capable of expanding SRCs in cord blood Lin ${ }^{-} \mathrm{CD} 34^{+} \mathrm{CD} 38^{-}$cells (16). Interestingly, after 4 days in the same culture conditions, SRCs in BM $\mathrm{Lin}^{-} \mathrm{CD} 34^{+} \mathrm{CD} 38^{-}$cells could not be expanded. The lack of SRC expansion after 4 days in culture under normoxic conditions suggests that human adult BM HSCs have different culture/cytokine requirements than cord blood HSCs. In contrast to normoxic conditions, we observed a significant increase in SRCs after 4 days in culture under $1.5 \% \mathrm{O}_{2}$. Not only were SRCs increased relative to normoxia, but we also observed a 4.2 -fold expansion of the number of SRCs in hypoxic cultures compared with freshly isolated $\mathrm{BM} \mathrm{Lin}{ }^{-} \mathrm{CD} 34^{+} \mathrm{CD} 38^{-}$ cells. These results demonstrate that low levels of $\mathrm{O}_{2}$ can greatly enhance the survival and/or self-renewal of human BM-repopulating cells in vitro. This finding is consistent with a previous report indicating that mouse BM-repopulating cells were better maintained in culture under severe hypoxia than were more committed progenitors (3). We could no longer detect SRC activity after 6 or 9 days in culture under $1.5 \%$ or $3 \%$ (data not shown) or $20 \% \mathrm{O}_{2}$. This finding indicates that, in the present culture conditions, which may not be optimal for BM stem cells, the positive effect of hypoxia on SRCs is short-lived.

The effect of hypoxia on clonogenic (CFC) and LTC-IC activity was limited to a reduction of granulocytic progenitors (G-CFU) and an increased number of LTC-ICs giving rise to BFU-E. The limited response of BM progenitors to hypoxia compared with the significant increase in SRCs observed in the same conditions demonstrates that the activity of human BM stem cells and progenitors is regulated differently by $\mathrm{O}_{2}$.
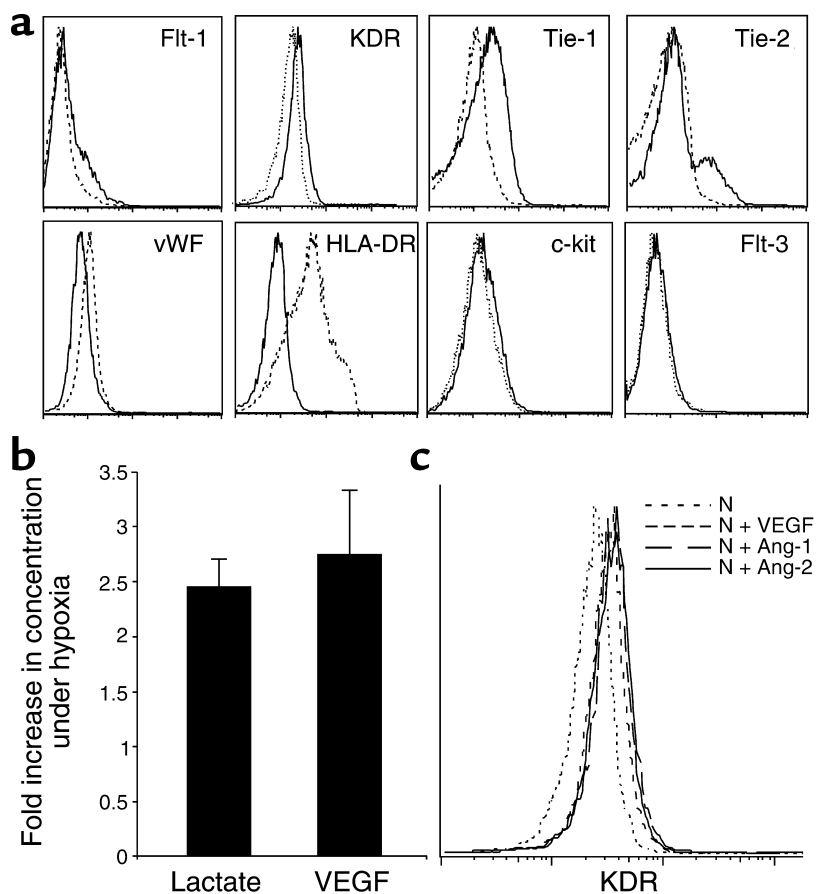

C

\section{Figure 5}

Hypoxia regulates the expression of HIF target genes in BM progenitors and stem cells. (a) Representative histograms of cell surface expression of angiogenic and hematopoietic cytokine receptors in BM $\mathrm{Lin}^{-} \mathrm{CD} 34^{+}$cells after 4 days in culture under normoxic (dashed lines) or hypoxic $\left(1.5 \% \mathrm{O}_{2}\right.$, solid lines) conditions. (b) The conditioned medium from BM Lin $-\mathrm{CD} 34^{+}$cells cultured overnight in normoxia or hypoxia $\left(1.5 \% \mathrm{O}_{2}\right)$ was assayed for lactate and human VEGF. Bars represent the fold increase in lactate and VEGF concentrations measured in hypoxic cultures relative to normoxic conditions. Error bars show SD. (c) Effect of VEGF, angiopoietin-1 (Ang-1), and angiopoietin-2 (Ang-2) on cell surface expression of VEGFR2 in Lin-CD34+ cells after 4 days in culture under normoxic conditions $(\mathrm{N})$. 
To further characterize the hypoxic response, we evaluated the effects of low $\mathrm{O}_{2}$ tension on the proliferation and cell cycle distribution of human BM progenitors and stem cells in vitro. The expansion of $\mathrm{Lin}^{-} \mathrm{CD} 34^{+}$ cells was slightly reduced at $1.5 \% \mathrm{O}_{2}$ compared with normoxia, indicating that most $\mathrm{BM}$ progenitors can survive and even proliferate under hypoxia. Interestingly, the expansion of purified $\mathrm{Lin}^{-} \mathrm{CD} 34^{+} \mathrm{CD} 38^{-}$cells was greater in hypoxic than in normoxic conditions. This positive effect of hypoxia on $\mathrm{Lin}^{-} \mathrm{CD} 34^{+} \mathrm{CD} 38$ cells demonstrates that primitive progenitors and stem cells are particularly well adapted to proliferating in a low- $\mathrm{O}_{2}$ environment.

To further evaluate the effect of hypoxia on the proliferation of BM cells, we used CFSE to track the division history of primitive subsets $\left(\mathrm{CD} 90^{+}, \mathrm{CXCR} 4^{+}\right.$, CD117 low, CD135 ${ }^{+}$, and HLA-DR ${ }^{-}$) of BM Lin ${ }^{-C D} 34^{+}$ cells cultured under hypoxia and normoxia. We show that under hypoxic conditions, the overall proliferative rates of $\mathrm{Lin}^{-} \mathrm{CD} 34^{+}$cells and subsets was slower in hypoxic conditions, resulting in fewer cells after 4 days in culture compared with normoxia. Hypoxia increased the percentage of the cells in $G_{0}$ or $G_{1}$ phase, while fewer cells were in found in $S$ or $G_{2} / M$. Furthermore, hypoxic conditions increased the proportion of cells in $\mathrm{G}_{1}$ phase relative to resting $\left(\mathrm{G}_{0}\right)$ cells, resulting in a dramatically decreased $G_{0}$ to $G_{1}$ ratio. This observation suggests that low $\mathrm{O}_{2}$ levels can either promote the transition from a resting state to $G_{1}$ phase and/or inhibit the transition from $G_{1}$ to $S$ phase in $B M$ progenitors and stem cells. It has been shown that hypoxia can induce $G_{1}$ arrest in tumor $(49,50)$ and endothelial cells (51). Gardner et al. (52) reported that, in fibroblasts, severe hypoxia can inhibit the $\mathrm{G}_{1} / \mathrm{S}$ transition through regulation of p27 expression. Wilpshaar et al. (53) found no difference in SCID-repopulating ability between uncultured cord blood $\mathrm{CD}^{3} 4^{+}$cells in $\mathrm{G}_{0}$ phase and those in $G_{1}$ phase. However, in cultured mobilized peripheral blood $\mathrm{CD}^{+} 4^{+}$cells, the level of human chimerism in NOD/SCID BM was lower in mice transplanted with cells in $\mathrm{G}_{1}$ phase compared with $\mathrm{G}_{0}(54)$. The expansion of SRCs we observed after 4 days in hypoxia and the increased number of cells in $G_{1}$ phase suggests that SRCs may be found in both $G_{0}$ and $\mathrm{G}_{1}$ phases under low oxygen levels.

In this study, we also investigated the cellular mechanisms involved in the hypoxic response of BM hematopoietic progenitor and stem cells. We found that HIF- $1 \alpha$ and ARNT are constitutively expressed in $\mathrm{Lin}^{-} \mathrm{CD} 34^{+} \mathrm{CD} 38^{-}$cells. However, HIF-2 $\alpha$ transcripts could not be detected in $\mathrm{Lin}^{-} \mathrm{CD} 34^{+} \mathrm{CD} 38^{-}$ cells. Our Western blot analysis of $\mathrm{Lin}^{-} \mathrm{CD} 34^{+}$cells shows that HIF- $1 \alpha$ protein is present under hypoxic conditions and is undetectable in normoxia. In contrast, ARNT protein is detectable regardless of $\mathrm{O}_{2}$ levels. This is consistent with previous reports showing that, in normoxic conditions, HIF- $1 \alpha$ is modified by a prolyl hydroxylase, an $\mathrm{O}_{2}$-dependent enzyme (55, $56)$. The modified form of HIF- $1 \alpha$ can interact with the von Hippel-Lindau tumor suppressor protein (VHL). This interaction induces the ubiquitination of HIF- $1 \alpha$ and its rapid degradation (57-60). Under hypoxic conditions, the hydroxylation no longer occurs, and HIF- $1 \alpha$ remains stable and can upregulate expression of its target genes.

Among the genes induced by hypoxia (25-29), angiogenic factors and receptors appear to play a critical role in the regulation of HSC survival and selfrenewal. In this report we show an increase in VEGF secretion and an upregulation of the cell surface expression of angiogenic receptors (Tie-2 and to a lesser extent VEGFR2 and Tie-1) under hypoxia. The hypoxia-induced increase in VEGF expression has been well characterized in other models (42-44). Recently, Gerber et al. (40) reported that VEGF regulates the survival of mouse HSCs through an internal autocrine loop. It has also been shown that human BM-repopulating cells express VEGF receptors $(61,62)$. It is thus tempting to speculate that a similar mechanism may be involved in regulating the survival of SRCs. Tie-2, the receptor for angiopoietin-1, has also been reported to be regulated by $\mathrm{O}_{2}$ levels (63) and can be found on HSCs (64). Thus, the combined effect of increased VEGF and enhanced responsiveness to angiogenic growth factors may play a role in the expansion of human BM-repopulating cells we observed. Future studies will be needed to further investigate the role of VEGF in the expansion of SRCs under hypoxia.

In conclusion, we show that the use of low $\mathrm{O}_{2}$ levels in vitro not only improves SRC survival compared with normoxia but also expands the number of human BM repopulating cells by 4.2 -fold compared with freshly isolated $\mathrm{Lin}^{-} \mathrm{CD} 34^{+} \mathrm{CD} 38^{-}$cells. The present report also establishes that hypoxia increases both VEGF secretions and responsiveness to angiogenic factors in BM progenitors and stem cells. In addition, we show that stem cells and progenitors respond differently to hypoxic conditions. These findings suggest that the balance between the survival, self-renewal, and differentiation of human BM HSCs might be tightly regulated by $\mathrm{O}_{2}$ levels. The beneficial effect of hypoxia on human BM-repopulating cells may be mediated by angiogenic factors such as VEGF. Finally, the use of low $\mathrm{O}_{2}$ tension could represent an important advance in the ex vivo expansion of human HSCs and may have important translational and clinical implications.

\section{Acknowledgments}

We are grateful to Jennifer Sinibaldi, Matthew Lessie, Amy Cleck, and Martin Carroll from the Stem Cell and Leukemia Core at the University of Pennsylvania Cancer Center for providing the large number of normal BM samples necessary for this study. The authors also thank William Demuth at the Abramson Family Cancer Research Center (University of Pennsylvania) for cell sorting, Tammi Coleman and Derrick Dow for their help with the NOD/SCID mouse colony, and Diana 
Ramirez-Bergeron, Andy Arsham, Brian Keith, and other members of the Simon Laboratory for their valuable input. M.C. Simon is an Investigator of the Howard Hughes Medical Institute.

1. Harrison, J.S., Rameshwar, P., Chang, V., and Bandari, P. 2002. Oxygen saturation in the bone marrow of healthy volunteers. Blood. 99:394.

2. Lichtman, M.A. 1981. The ultrastructure of the hemopoietic environment of the marrow: a review. Exp. Hematol. 9:391-410.

3. Cipolleschi, M.G., Dello Sbarba, P., and Olivotto, M. 1993. The role of hypoxia in the maintenance of hematopoietic stem cells. Blood. 82:2031-2037.

4. Ivanovic, Z., et al. 2000. Incubation of murine bone marrow cells in hypoxia ensures the maintenance of marrow-repopulating ability together with the expansion of committed progenitors. Br. J. Haematol. 108:424-429.

5. Adelman, D.M., Maltepe, E., and Simon, M.C. 1999. Multilineage embryonic hematopoiesis requires hypoxic ARNT activity. Genes Dev. 13:2478-2483.

6. Cipolleschi, M.G., et al. 1997. Severe hypoxia enhances the formation of erythroid bursts from human cord blood cells and the maintenance of BFU-E in vitro. Exp. Hematol. 25:1187-1194.

7. Quinlan, D.P., Jr., et al. 1998. Effect of hypoxia on the hematopoietic and immune modulator preprotachykinin-I. Arch. Surg. 133:1328-1334.

8. Ivanovic, Z., Dello Sbarba, P., Trimoreau, F., Faucher, J.L., and Praloran, V. 2000. Primitive human HPCs are better maintained and expanded in vitro at 1 percent oxygen than at 20 percent. Transfusion. 40:1482-1488.

9. Dick, J.E. 1996. Normal and leukemic human stem cells assayed in SCID mice. Semin. Immunol. 8:197-206.

10. Larochelle, A., et al. 1996. Identification of primitive human hematopoietic cells capable of repopulating NOD/SCID mouse bone marrow: implications for gene therapy. Nat. Med. 2:1329-1337.

11. Pflumio, F., et al. 1996. Phenotype and function of human hematopoietic cells engrafting immune-deficient CB17-severe combined immunodeficiency mice and nonobese diabetic-severe combined immunodeficiency mice after transplantation of human cord blood mononuclear cells. Blood. 88:3731-3740.

12. Bhatia, M., Wang, J.C.Y., Kapp, U., Bonnet, D., and Dick, J.E. 1997. Purification of primitive human hematopoietic cells capable of repopulating immune-deficient mice. Proc. Natl. Acad. Sci. U. S. A. 94:5320-5325.

13. Wang, J.C., Doedens, M., and Dick, J.E. 1997. Primitive human hematopoietic cells are enriched in cord blood compared with adult bone marrow or mobilized peripheral blood as measured by the quantitative in vivo SCID-repopulating cell assay. Blood. 89:3919-3924.

14. Holyoake, T.L., Nicolini, F.E., and Eaves, C.J. 1999. Functional differences between transplantable human hematopoietic stem cells from fetal liver, cord blood, and adult marrow. Exp. Hematol. 27:1418-1427.

15. Danet, G.H., Lee, H.W., Luongo, J.L., Simon, M.C., and Bonnet, D.A. 2001. Dissociation between stem cell phenotype and NOD/SCID repopulating activity in human peripheral blood CD34(+) cells after ex vivo expansion. Exp. Hematol. 29:1465-1473.

16. Bhatia, M., et al. 1997. Quantitative analysis reveals expansion of human hematopoietic repopulating cells after short-term ex vivo culture. J. Exp. Med. 186:619-624.

17. Conneally, E., Cashman, J., Petzer, A., and Eaves, C. 1997. Expansion in vitro of transplantable human cord blood stem cells demonstrated using a quantitative assay of their lympho-myeloid repopulating activity in nonobese diabetic-scid/scid mice. Proc. Natl. Acad. Sci. U. S. A. 94:9836-9841.

18. Piacibello, W., et al. 1999. Engraftment in nonobese diabetic severe combined immunodeficient mice of human CD34(+) cord blood cells after ex vivo expansion: evidence for the amplification and self-renewal of repopulating stem cells. Blood. 93:3736-3749.

19. Bhatia, M., et al. 1999. Bone morphogenetic proteins regulate the developmental program of human hematopoietic stem cells. J. Exp. Med. 189:1139-1148.

20. Ueda, T., et al. 2000. Expansion of human NOD/SCID-repopulating cells by stem cell factor, Flk2/Flt3 ligand, thrombopoietin, IL-6, and soluble IL-6 receptor. J. Clin. Invest. 105:1013-1021.

21. Bryder, D., and Jacobsen, S.E. 2000. Interleukin-3 supports expansion of long-term multilineage repopulating activity after multiple stem cell divisions in vitro. Blood. 96:1748-1755.

22. Kollet, O., et al. 2000. The plant lectin FRIL supports prolonged in vitro maintenance of quiescent human cord blood CD34(+)CD38 (-/low)/SCID repopulating stem cells. Exp. Hematol. 28:726-736.

23. Shih, C.C., et al. 2000. A secreted and LIF-mediated stromal cell-derived activity that promotes ex vivo expansion of human hematopoietic stem cells. Blood. 95:1957-1966.

24. Chute, J.P., et al. 2002. Ex vivo culture with human brain endothelial cells increases the SCID-repopulating capacity of adult human bone marrow. Blood. 100:4433-4439.

25. Harris, A.L. 2002. Hypoxia-a key regulatory factor in tumour growth. Nat. Rev. Cancer. 2:38-47.

26. Kaelin, W.G., Jr. 2002. How oxygen makes its presence felt. Genes Dev. 16:1441-1445.

27. Wenger, R.H. 2002. Cellular adaptation to hypoxia: O2-sensing protein hydroxylases, hypoxia-inducible transcription factors, and $\mathrm{O} 2$-regulated gene expression. FASEB J. 16:1151-1162.

28. Semenza, G.L. 2001. HIF-1 and mechanisms of hypoxia sensing. Curr. Opin. Cell Biol. 13:167-171.

29. Ramirez-Bergeron, D.L., and Simon, M.C. 2001. Hypoxia-inducible factor and the development of stem cells of the cardiovascular system. Stem Cells. 19:279-286.

30. Jiang, B.H., Semenza, G.L., Bauer, C., and Marti, H.H. 1996. Hypoxiainducible factor 1 levels vary exponentially over a physiologically relevant range of $\mathrm{O} 2$ tension. Am. J. Physiol. 271:C1172-C1180.

31. Semenza, G.L. 1998. Hypoxia-inducible factor 1 and the molecular physiology of oxygen homeostasis. J. Lab. Clin. Med. 131:207-214.

32. Tian, H., McKnight, S.L., and Russell, D.W. 1997. Endothelial PAS domain protein 1 (EPAS1), a transcription factor selectively expressed in endothelial cells. Genes Dev. 11:72-82.

33. Ema, M., et al. 1997. A novel bHLH-PAS factor with close sequence similarity to hypoxia-inducible factor 1alpha regulates the VEGF expression and is potentially involved in lung and vascular development. Proc. Natl. Acad. Sci. U. S. A. 94:4273-4278.

34. Flamme, I., et al. 1997. HRF, a putative basic helix-loop-helix-PASdomain transcription factor is closely related to hypoxia-inducible factor-1 alpha and developmentally expressed in blood vessels. Mech. Dev. 63:51-60.

35. Gu, Y.Z., Moran, S.M., Hogenesch, J.B., Wartman, L., and Bradfield, C.A. 1998. Molecular characterization and chromosomal localization of a third alpha-class hypoxia inducible factor subunit, HIF3alpha. Gene Expr. 7:205-213.

36. Guzman, M.L., et al. 2001. Nuclear factor-kappaB is constitutively activated in primitive human acute myelogenous leukemia cells. Blood. 98:2301-2307.

37. Hogge, D.E., Lansdorp, P.M., Reid, D., Gerhard, B., and Eaves, C.J. 1996. Enhanced detection, maintenance, and differentiation of primitive human hematopoietic cells in cultures containing murine fibroblasts engineered to produce human steel factor, interleukin-3, and granulocyte colony-stimulating factor. Blood. 88:3765-3773.

38. Sutherland, H.J., Eaves, C.J., Lansdorp, P.M., Thacker, J.D., and Hogge, D.E. 1991. Differential regulation of primitive human hematopoietic cells in long-term cultures maintained on genetically engineered murine stromal cells. Blood. 78:666-672.

39. Bonnet, D., Bhatia, M., Wang, J.C., Kapp, U., and Dick, J.E. 1999. Cytokine treatment or accessory cells are required to initiate engraftment of purified primitive human hematopoietic cells transplanted at limiting doses into NOD/SCID mice. Bone Marrow Transplant. 23:203-209.

40. Gerber, H.P., et al. 2002. VEGF regulates haematopoietic stem cell survival by an internal autocrine loop mechanism. Nature. 417:954-958.

41. Hattori, K., et al. 2001. Vascular endothelial growth factor and angiopoietin-1 stimulate postnatal hematopoiesis by recruitment of vasculogenic and hematopoietic stem cells. J. Exp. Med. 193:1005-1014.

42. Liu, Y., Cox, S.R., Morita, T., and Kourembanas, S. 1995. Hypoxia regulates vascular endothelial growth factor gene expression in endothelial cells. Identification of a $5^{\prime}$ enhancer. Circ. Res. 77:638-643.

43. Forsythe, J.A., et al. 1996. Activation of vascular endothelial growth factor gene transcription by hypoxia-inducible factor $1 . \mathrm{Mol}$. Cell. Biol. 16:4604-4613.

44. Levy, A.P., Levy, N.S., Wegner, S., and Goldberg, M.A. 1995. Transcriptional regulation of the rat vascular endothelial growth factor gene by hypoxia. J. Biol. Chem. 270:13333-13340.

45. Wang, G.L., and Semenza, G.L. 1993. Characterization of hypoxiainducible factor 1 and regulation of DNA binding activity by hypoxia. J. Biol. Chem. 268:21513-21518.

46. Semenza, G.L., Roth, P.H., Fang, H.M., and Wang, G.L. 1994. Transcriptional regulation of genes encoding glycolytic enzymes by hypoxia-inducible factor 1. J. Biol. Chem. 269:23757-23763.

47. Firth, J.D., Ebert, B.L., Pugh, C.W., and Ratcliffe, P.J. 1994. Oxygen-regulated control elements in the phosphoglycerate kinase 1 and lactate dehydrogenase A genes: similarities with the erythropoietin $3^{\prime}$ enhancer. Proc. Natl. Acad. Sci. U. S. A. 91:6496-6500.

48. Firth, J.D., Ebert, B.L., and Ratcliffe, P.J. 1995. Hypoxic regulation of lactate dehydrogenase A. Interaction between hypoxia-inducible factor 1 and cAMP response elements. J. Biol. Chem. 270:21021-21027.

49. Graeber, T.G., et al. 1996. Hypoxia-mediated selection of cells with diminished apoptotic potential in solid tumours. Nature. 379:88-91. 50. Schmaltz, C., Hardenbergh, P.H., Wells, A., and Fisher, D.E. 1998. Reg- 
ulation of proliferation-survival decisions during tumor cell hypoxia. Mol. Cell. Biol. 18:2845-2854.

51. Iida, T., et al. 2002. Hypoxia-inducible factor-1alpha induces cell cycle arrest of endothelial cells. Genes Cells. 7:143-149.

52. Gardner, L.B., et al. 2001. Hypoxia inhibits G1/S transition through regulation of p27 expression. J. Biol. Chem. 276:7919-7926.

53. Wilpshaar, J., et al. 2000. Similar repopulating capacity of mitotically active and resting umbilical cord blood CD34 $(+)$ cells in NOD/SCID mice. Blood. 96:2100-2107.

54. Gothot, A., van der Loo, J.C., Clapp, D.W., and Srour, E.F. 1998. Cell cycle-related changes in repopulating capacity of human mobilized peripheral blood CD34(+) cells in non-obese diabetic/severe combined immune-deficient mice. Blood. 92:2641-2649.

55. Jaakkola, P., et al. 2001. Targeting of HIF-alpha to the von Hippel-Lindau ubiquitylation complex by O2-regulated prolyl hydroxylation. Science. 292:468-472.

56. Ivan, M., et al. 2001. HIFalpha targeted for VHL-mediated destruction by proline hydroxylation: implications for $\mathrm{O} 2$ sensing. Science. 292:464-468.

57. Cockman, M.E., et al. 2000. Hypoxia inducible factor-alpha binding and ubiquitylation by the von Hippel-Lindau tumor suppressor protein.
J. Biol. Chem. 275:25733-25741.

58. Ohh, M., et al. 2000. Ubiquitination of hypoxia-inducible factor requires direct binding to the beta-domain of the von Hippel-Lindau protein. Nat. Cell Biol. 2:423-427.

59. Tanimoto, K., Makino, Y., Pereira, T., and Poellinger, L. 2000. Mechanism of regulation of the hypoxia-inducible factor- 1 alpha by the von Hippel-Lindau tumor suppressor protein. EMBO J. 19:4298-4309.

60. Kamura, T., et al. 2000. Activation of HIF1alpha ubiquitination by a reconstituted von Hippel-Lindau (VHL) tumor suppressor complex. Proc. Natl. Acad. Sci. U. S. A. 97:10430-10435.

61. Ziegler, B.L., et al. 1999. KDR receptor: a key marker defining hematopoietic stem cells. Science. 285:1553-1558.

62. Hattori, K., et al. 2002. Placental growth factor reconstitutes hematopoiesis by recruiting VEGFR1(+) stem cells from bone-marrow microenvironment. Nat. Med. 8:841-849.

63. Oh, H., et al. 1999. Hypoxia and vascular endothelial growth factor selectively up-regulate angiopoietin- 2 in bovine microvascular endothelial cells. J. Biol. Chem. 274:15732-15739.

64. Hashiyama, M., et al. 1996. Predominant expression of a receptor tyrosine kinase, TIE, in hematopoietic stem cells and B cells. Blood. 87:93-101. 